\title{
HIERARCHICAL STRUCTURE IN
}

\section{DARK MATTER DISTRIBUTIONS}

\author{
K. MAKISHIMA
}

Department of Physics, University of Tokyo

7-9-1 Hongo, Bunkyo-ku, Tokyo, Japan 113

\section{Introduction and Summary}

Galaxy observers argue for the dark halo surrounding each galaxy, while cluster investigators discuss the dominance of dark matter (DM) in the intra-cluster space. Then, what is the relation between the cluster DM and the DM associated with member galaxies of the cluster?

$A S C A$ studies of $\mathrm{cD}$ clusters ( $\$ 2)$ have shown that, while a cluster has a massive dark halo, the $\mathrm{CD}$ galaxy also has its own smaller-scale dark halo, thus making a hierarchical halo-in-halo structure. Such a nested potential structure has also been discovered around several X-ray bright non-cD elliptical galaxies (\$3).

\section{Clusters with cD Galaxies}

As has long been known (e.g. Jones \& Forman 1984), some clusters of galaxies, particularly those with $\mathrm{cD}$ galaxies, exhibit centrally peaked $\mathrm{X}$ ray surface brightness profiles with small (e.g. $<100 \mathrm{kpc}$ ) X-ray core radii; others exhibit flatter X-ray profiles with larger core radii.

In the former type of objects, the ICM is thought to be cooling significantly and becoming very dense in the central regions, because of the short cooling time. The centrally peaked X-ray emissivities observed from many cD clusters are usually explained in terms of such cooling effects.

As an alternative possibility, the X-ray emissivity may be enhanced in the core region of a $\mathrm{cD}$ cluster because the gravitational potential associated with the $\mathrm{cD}$ galaxy attracts excess ICM. Although this simple-minded interpretation so far received little attention, this has actually been confirmed to be the case with many $\mathrm{cD}$ clusters through $A S C A$ studies. 
TABLE 1. Parameters of the central excess X-ray emission $\left(h_{0}=75\right)$.

\begin{tabular}{lllllll}
\hline object & $\begin{array}{l}k T^{a)} \\
(\mathrm{keV})\end{array}$ & $\begin{array}{l}R_{\text {excess }}^{b)} \\
(\mathrm{kpc})\end{array}$ & $\begin{array}{l}M_{\text {excess }}^{c)} \\
\left(M_{\odot}\right)\end{array}$ & $\begin{array}{l}L_{\text {excess }}^{0.5-10} \\
\left(\mathrm{erg} \mathrm{s}^{-1}\right)\end{array}$ & $\begin{array}{l}L_{\text {cool }}^{0.5-10} \\
\left(\mathrm{erg} \mathrm{s}^{-1}\right)\end{array}$ & $\mathrm{M} / \mathrm{L}$ \\
\hline NGC 4636 & 0.9 & 25 & $6 \times 10^{11}$ & $1.5 \times 10^{41}$ & - & 20 \\
Fornax & 1.2 & 60 & $2 \times 10^{12}$ & $1.6 \times 10^{42}$ & - & 25 \\
Centaurus & 3.8 & 70 & $3 \times 10^{12}$ & $1 \times 10^{43}$ & $4 \times 10^{42}$ & 60 \\
Hydra-A & 3.5 & 60 & $5 \times 10^{12}$ & $8 \times 10^{43}$ & $3 \times 10^{42}$ & 45 \\
A1795 & 6.1 & 100 & $1.5 \times 10^{13}$ & $2 \times 10^{44}$ & $6 \times 10^{43}$ & 50 \\
\hline
\end{tabular}

a) Average ICM temperature excluding the central region.

b) The radius within which the excess $\mathrm{X}$-ray emission is seen.

b) The excess total gravitating mass inside $R_{\text {excess }}$.

\subsection{THE FORNAX CLUSTER}

The Fornax cluster is the first example in which the central excess X-ray emission was confirmed to arise due to the potential well of the $\mathrm{cD}$ galaxy, NGC 1399 (Ikebe et al. 1996). Details are summarized as follows.

a. The X-ray surface brightness profile exhibits a clear excess around the $\mathrm{cD}$ galaxy, above a single $\beta$-model fitted to the outer region.

b. The overall $X$-ray emission is approximately isothermal within $\sim 20 \%$.

c. The X-ray profile can be described by a sum of two projected $\beta$-model components, which cross over at a radius $R_{\text {excess }}$ given in Table 1 .

$\mathrm{d}$. The $\mathrm{X}$-ray volume emissivity can be modeled as a quadrature sum of the two deprojected $\beta$-model components.

e. The spherically-integrated total mass curve $M(R)$ exhibits a clear hierarchy, corresponding to the $\mathrm{cD}$ galaxy and the cluster.

f. The hierarchy exists not only in the total mass but also in the DM mass, estimated by removing the stellar and ICM masses from $M(R)$.

\subsection{THE HYDRA-A CLUSTER AND A1795}

Similar results as described above have been obtained from two prototypical "cooling flow" clusters; the Hydra-A cluster by Ikebe et al. (1997a), and A1795 by Xu et al. (1997). Using the ROSAT and ASCA data, these authors revealed that the prominent central excess $\mathrm{X}$-ray emission of these clusters are detectable not only in soft X-rays but also in hard X-rays up to $10 \mathrm{keV}$. Items a. $-\mathrm{f}$. listed in $\$ 2.1$ apply to these two clusters as well. Furthermore, they commonly exhibit the following two additional properties.

g. An additional cool emission is seen near the cluster center, but its luminosity $L_{\text {cool }}$ does not account for the excess emission (Table 1). 
h. The cooling-flow rates estimated with $A S C A$ fall several times short of those estimated previously without the knowledge of hard X-ray data.

Based on $N$-body simulations, Navarro et al. (1996) propose a universal shape of the DM distribution, which exhibits a central cusp as compared to the classical King-type potential. However, the central excess emission of A1795 is too strong to be explained by the "universal halo" profile.

\subsection{THE CENTAURUS CLUSTER}

The Centaurus cluster shows a very strong central cool emission, and the most prominent central metallicity increase ever measured from clusters (Fukazawa et al. 1994; Ikebe et al. 1997b). However even in this case, the central cluster volume is mostly filled with the hot ICM in an apparent twophase configuration, and the central excess is seen in hard X-ray continuum as well. Therefore the centrally-peaked X-ray profile of this cluster must be caused, at least partially, by the central excess potential associated with the $\mathrm{cD}$ galaxy, NGC 4696 (Ikebe et al. 1997b).

Within $\sim 50 \mathrm{kpc}$ of the center, there take place the three outstanding phenomena of the Centaurus cluster; the cool emission component, the metallicity increase, and the excess hard X-ray emissivity. All these phenomena are probably caused by the $\mathrm{CD}$ galaxy, in such a way that the metal-enriched cool inter-stellar medium of NGC 4696 is confined by its own potential plus the external ICM pressure.

\section{Elliptical galaxies}

Matsushita (1997) and Matsushita et al. (1997a) have discovered that elliptical galaxies can be classified into two subgroups in terms of their X-ray properties. One (the other) class of objects are characterized by a high (low) $\mathrm{X}$-ray luminosity, a high (low) ISM metallicity, and the X-ray emission extending (not extending) beyond the optical galaxy.

The giant elliptical galaxy NGC 4636 is the best example of the X-ray extended class of objects. From a deep ( $200 \mathrm{ks}) A S C A$ exposure, the radial $\mathrm{X}$-ray brightness profile of NGC 4636 was found to consist of two $\beta$-model components (Matsushita 1997; Matsushita et al. 1997b). The subsequent story (Fig.1) is essentially the same as that of the Fornax cluster ( $\$ 2.1$ ). However in the case of NGC 4636, the mass component corresponding to the larger $\beta$-model component, with $M / L \sim 200$, has no clear counterpart in the visible light. It is hence suggested that NGC 4636 sits at the bottom of a group of galaxies that is optically invisible. Other X-ray luminous noncD elliptical galaxies are also inferred to signal the presence of a group-size $\mathrm{DM}$ concentration, with little optical indication. 


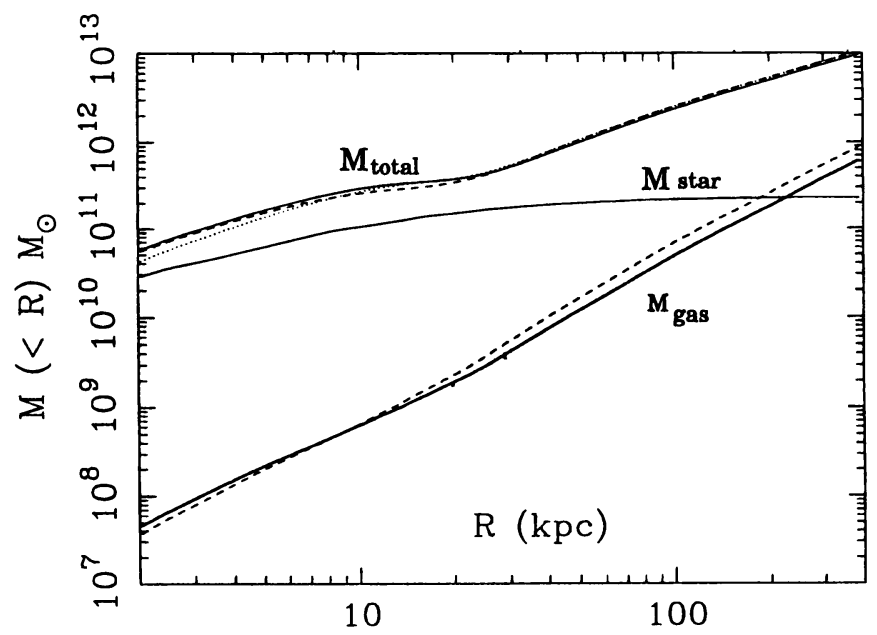

Figure 1. The spherically integrated mass profiles of NGC 4636 (Matsushita 1997).

\section{Discussion and Implication}

When the X-ray emitting hot gas confined in a self-gravitating system exhibits a deviation from a single- $\beta$ distribution, there is a high probability that the underlying gravitational potential has a nested halo-in-halo structure, which in turn reflects a hierarchy in the dark matter distribution. These effects are seen over a wide range of system richness, including ellipticals (§3), groups (Mulchaey \& Zabludoff 1997), and clusters (\$2).

The double- $\beta$ model has been found to be very useful; its application to the $\mathrm{X}$-ray data analysis is strongly encouraged. When a single- $\beta$ profile is forced to fit a brightness profile that is intrinsically of double- $\beta$ nature, the core radius and $\beta$ both tend to get smaller. This effect can nicely account for the apparent positive correlation between these two quantities observed from a large ROSAT sample of clusters (Pownall \& Stewart 1995).

\section{References}

Fukazawa, Y., et al. (1994), Publ. Astr. Soc. Japan 46, L55

Pownall, H. R., \& Stewart, G. C. (1996), in Röntgenstrahlung from the Universe.

Ikebe, Y., et al. (1996), Nature, 379, 427

Ikebe, Y., et al. (1997a), Astrophys. J., 481, 660

Ikebe, Y., et al. (1997b), submitted to Astrophys. J.; see also this volume.

Jones, C., \& Forman, W. (1984), Astrophys. J., 276, 38

Mulchaey, J. S., \& Zabludoff, A. I. (1997), Astrophys. J., in press

Matsushita, K. (1997), PhD Thesis, University of Tokyo

Matsushita, K., et al. (1997a), Astrophys. J., in press; see also this volume.

Matsushita, K., et al. (1997b), submitted to Nature; see also this volume.

Navarro, J. F, Frenk, C. S., \& White, S. D. M. (1996), Astrophys. J., 462, 563

$\mathrm{Xu}, \mathrm{H}$., et al. (1997), submitted to Astrophys. J.; see also this volume. 